\title{
Computer-Assisted Soft-Tissue Surgery Training and Monitoring
}

\author{
M.P.S.F. Gomes, A.R.W. Barrett, and B.L. Davies \\ Mechatronics in Medicine Laboratory, Department of Mechanical Engineering, \\ Imperial College of Science, Technology and Medicine, Exhibition Road, \\ London SW7 2BX, UK \\ \{p.gomes, a.r.w.barrett, b.davies\}@ic.ac.uk \\ http://www.me.ic.ac.uk/case/mim/
}

\begin{abstract}
Last year at MICCAI, a computer-assisted surgical training system (CASTS) for Transurethral Resection of the Prostate (TURP), was presented [1]. Unpredictable deformation of the prostate phantom caused unacceptable inaccuracies. Solutions have now been investigated which are described in this paper, namely constraining the phantom, using a different phantom, and tracking the phantom's deformation and movement with ultrasound. Ethics Committee approval was obtained to assess the feasibility of using CASTS as an in vivo system for surgical monitoring (CASMS). Pre-operative ultrasound of the patient forms the basis of the computer-generated prostate's model. Peroperative ultrasound is employed to detect and compensate for prostate movement. An optically tracked ultrasound probe is used to relate the images to the optically tracked resectoscope's coordinate system. The CASMS in vivo system will help in the assessment of completeness of resection, the provision of information of resection both distal to the verumontanum and near to the capsule. Tests of the CASMS system in the operating room are described.
\end{abstract}

\section{Introduction}

In CASTS, the surgeon resects a prostate phantom using real surgical tools (optically tracked), under endoscopic visual guidance (Fig.1). An additional computer display (Fig.2) provides navigational information and shows the progression of the resection.

Whilst evaluating CASTS, it was found that the prostate phantom moved and deformed significantly. This movement has to be taken into account and the graphical model suitably updated. Per-operative ultrasound has been suggested for this and is being evaluated in the laboratory using specially developed gelatine prostate phantoms (Fig.3). Preliminary results are presented in this paper.

The extension of the trainer to the operating room, to be used as an in vivo monitoring system (CASMS), is now underway and some results are discussed. This novel two-stage approach aims to bridge the gap between training on a simulator and performing a real surgical procedure.

In CASMS (Fig.4), the surgeon acquires a set of 2D pre-operative transrectal ultrasound scans of the patient's prostate, using an optically tracked ultrasound probe. The scans are then manually segmented by the urologist who delineates the areas to be resected. A 3D model is automatically built and rendered in a computer display.

W. Niessen and M. Viergever (Eds.): MICCAI 2001, LNCS 2208, pp. 360-367, 2001.

(C) Springer-Verlag Berlin Heidelberg 2001 
During the TURP, the display shows the current status of the resection by superimposing a rendering of the resectoscope onto the patient's prostate model. The resected cavity is also rendered. Visual warnings are given if the tip of the resectoscope is outside the resectable volume.

Despite the prostate being anchored to the adjacent anatomical structures, some degree of movement is also to be expected. Per-operative ultrasound can be used to detect and compensate for this movement.

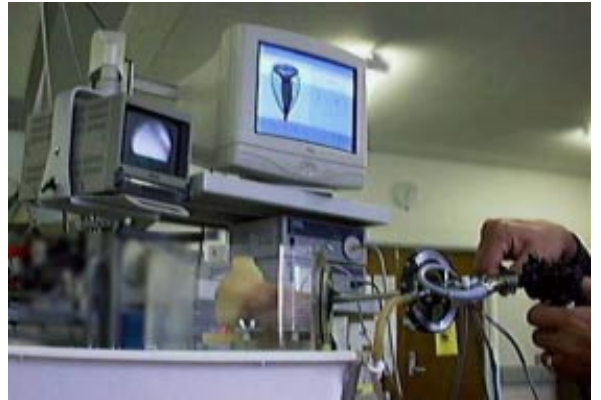

Fig. 1. CASTS in use

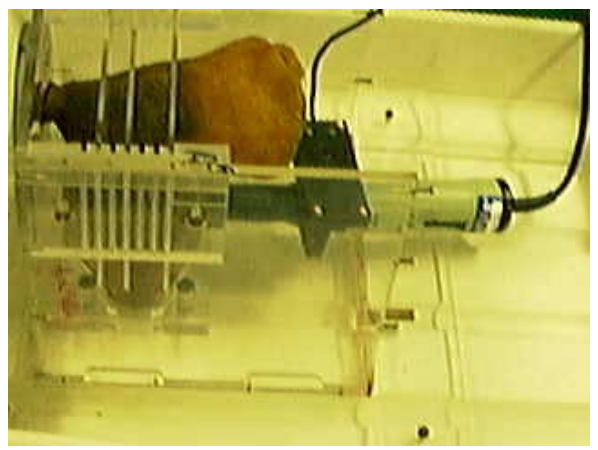

Fig. 3. Perspex profiles and ultrasound

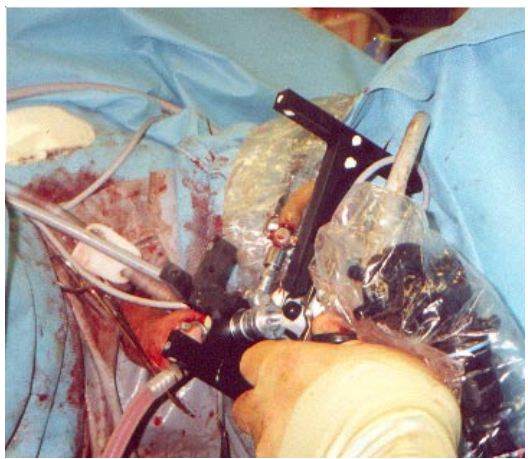

Fig. 5. Mock-up tools in use in the o.r.

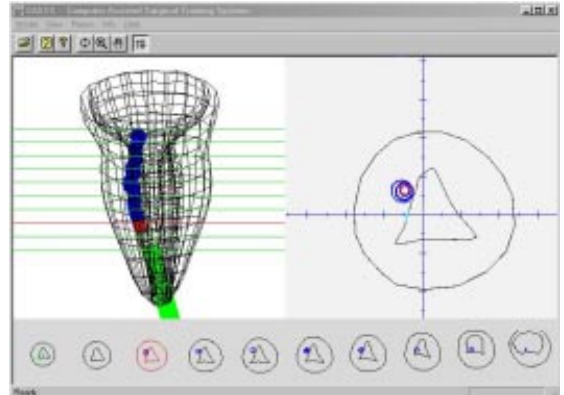

Fig. 2. Graphical User Interface

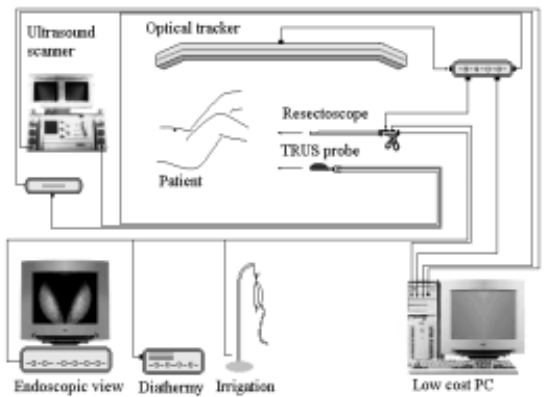

Fig. 4. The in vivo monitoring system

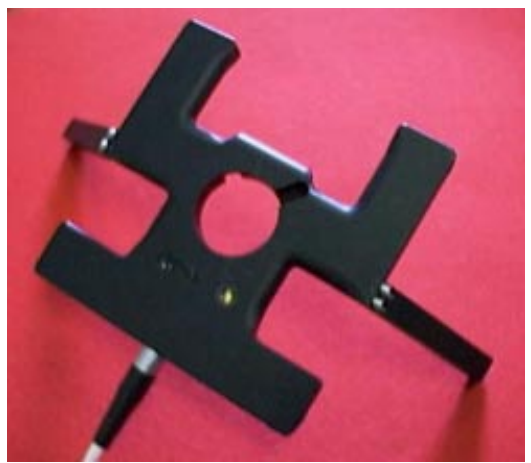

Fig. 6. IRED tool (by Traxtal Technologies) 
Mock-ups of instrumentation of the resectoscope have already been tried in the operating room and were found not to interfere with the procedure (Fig.5). Sterilisable tools have been manufactured (Fig.6).

\section{Materials and Methods}

\section{Constraints to Phantom Movement}

One of the problems encountered with the TURP trainer was the deformation of the phantom due to cutting and due to absorption of the irrigating fluic ${ }^{1}$ during resection. Also, the forces exerted by the resectoscope on the phantom caused it to move and deform significantly. Tracking the phantom with the optical tracker was not deemed a practical solution as it would interfere with the cutting, and because the phantom is not a rigid body.

A solution that partly solved the problem was the introduction of physical constraints. A set of Perspex profiles was built to limit the severity of the displacements and deformations (Fig.3). Complete constraint of the phantom would be unrealistic and would restrict the movement of the resectoscope, preventing complete resection. Therefore, some degree of motion has to be allowed which must then be detected and compensated for.

\section{Prostate Phantoms - The Resectable Volume}

It was decided to investigate the use of ultrasound to track the phantom's (and subsequently the patient's) prostate. Since the phantom being used (Limbs \& Things, Bristol, UK) is opaque to ultrasound, an alternative had to be found.

A mould for gelatine phantoms was designed, based on a resection shape from anatomical diagrams [2]. The phantom has a channel, simulating the urethra, that allows the introduction of a resectoscope and subsequent resection. The phantom is inserted into a Perspex box which is filled with gelatine (Figs.7,8). Another channel, simulating the rectum, allows the insertion of an ultrasound probe. Wires may be strung across the phantom so that the ultrasound scans can be acquired in known planes. Replaceable sides on the Perspex box allow the phantom to be cut into slices corresponding to the ultrasound planes.

\section{Prostate Phantoms - The Modified Limbs \& Things}

The initial design of the phantom contained a straight narrow urethra, which constrained the resectoscope to making shallow cuts, so that the whole volume could not be resected. It was decided to construct a second phantom based on the shape of the Limbs and Things model used in the CASTS trainer system, with an additional base section to allow insertion of the ultrasound probe. The Limbs and Things phantom models the whole of the prostate rather than just the resectable volume and

\footnotetext{
${ }^{1}$ The irrigant for the phantom resections was water.
} 
includes anatomical features such as the verumontanum and bladder neck, thus allowing a laboratory based assessment of the in vivo system, more closely mimicking the actual operation.

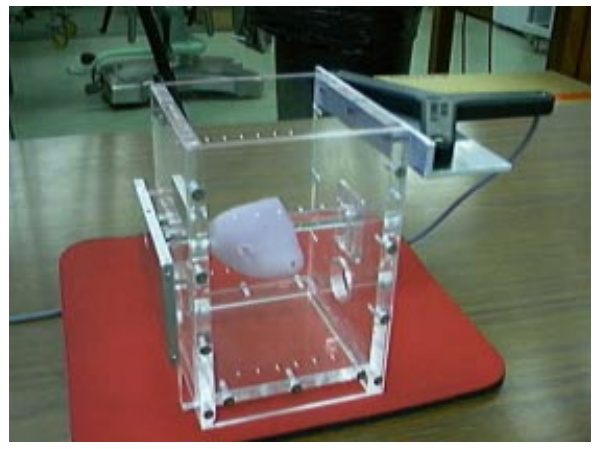

Fig. 7. A wax cast of the prostate phantom inserted into the Perspex box. Two Perspex rods, one through the cast, one below, mark the locations of the simulated urethra and rectum.

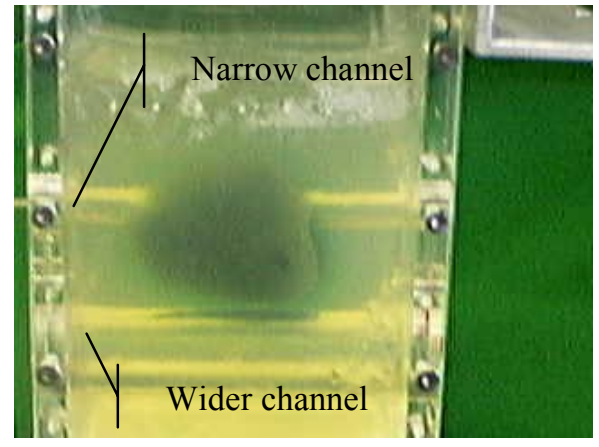

Fig. 8. The gelatine prostate in a gelatine bath. The narrow channel through the prostate simulates the urethra. The wider channel below the prostate simulates the rectum.

Formers and moulds for creating the phantom are shown in Figure 9. Three moulds are required for making the phantom. The formers $(a, b, c)$ were provided by Limbs and Things. The first mould (d) models the void of the urethra and bladder neck. This mould is created in two halves by masking off half the former (a) using clay, pouring over silicone rubber which is left to cure, then removing the clay and pouring the second half. A latex skin (Fig.10a) is created by repeated filling of the mould with latex, pouring out the excess and allowing each layer to cure. The second and third moulds (f,g) are made similarly, and model the prostate capsule and surrounding matrix respectively.

The latex skin is filled with water and frozen. The latex skin is then placed in the second mould (e) and gelatine solution is poured into the mould. The solution is $18 \%$ gelatine powder (Supercook, Leeds, England) and 9\% psyllium husk powder (Regucol, Holland \& Barrett Ltd, USA) by weight. The psyllium husk powder scatters the ultrasound beam giving an ultrasound image similar to human tissue [3].

Once set, the gelatine prostate is placed in the third mould which is filled with gelatine solution excluding the psyllium powder. Food colourings are added to the gelatine mixtures to visually distinguish the prostate from its surrounds. Once set, the ice is melted from the latex skin and the skin removed.

The finished phantom is placed inside a latex bladder (Fig.10b) to provide stability and reduce the likelihood of the phantom tearing on deformation. The bladder also provides a reservoir for irrigation fluid to collect, thus ensuring that the urethra is always filled so the ultrasound beam can penetrate the top surface of the phantom. Figure 3 shows the phantom and bladder constrained with Perspex plates and ready for resection. 

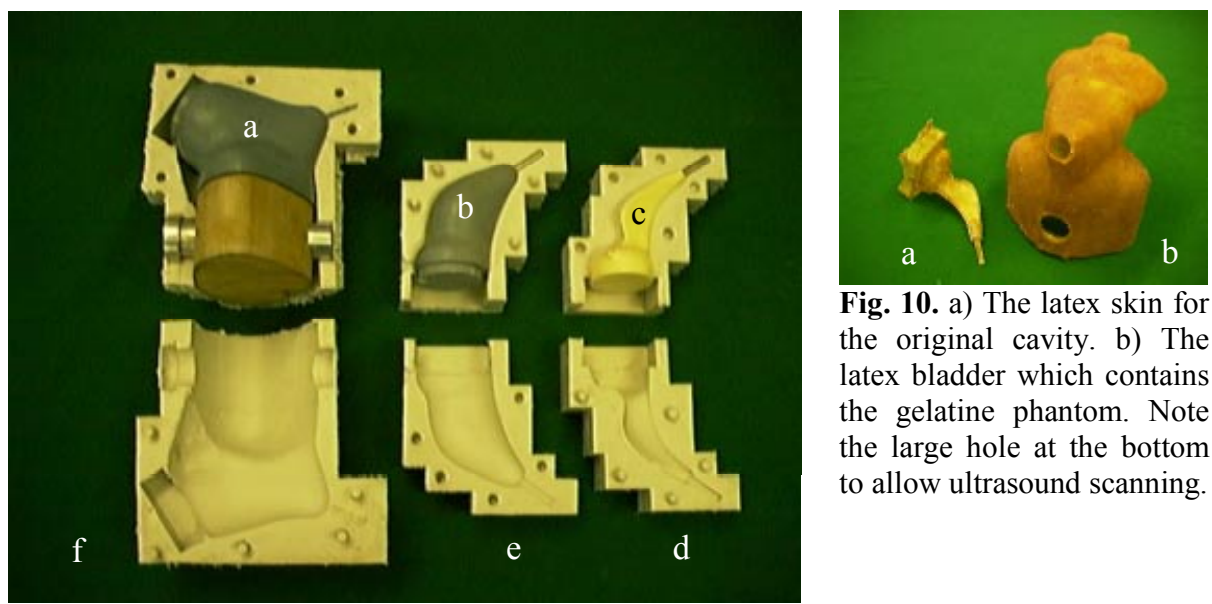

Fig. 10. a) The latex skin for the original cavity. b) The latex bladder which contains the gelatine phantom. Note the large hole at the bottom to allow ultrasound scanning.

Fig. 9. Formers and moulds for the gelatine phantom

\section{Prostate Modelling from Ultrasound}

The procedure for generating a patient-specific prostate model is now described.

The surgeon identifies the limits of resection along the urethra by marking points using the tracked resectoscope. Using a tracked transrectal probe, a series of ultrasound scans with known position and orientation is collected which intersect the resectable region. The surgeon demarcates the region of resection within each scan. Scans may be marked in two ways - with a complete outline if the surgeon can clearly see the resectable region, or a segmented outline if only parts of the region are visible. The complete outline is calculated by spline interpolation through a small number of supplied points.

The outline data consists of a set of points (directly from the segments or interpolated from the complete outlines) lying on the resection volume boundary in a cylindrical coordinate system (linear along the line of the urethra and radial perpendicular to this line). The data is mapped to a $2 \mathrm{D}$ data space by assuming that the radius of the points does not vary much over the interpolation range (which is valid for a smoothly varying shape such as the prostate) and unwrapping the cylindrical system onto a flat plane. A 2D Delauney triangulation algorithm is used to construct a triangular mesh with added constraints to ensure that triangles are not created from three points which derive from the same ultrasound slice. The radius of any point on the resection volume surface may be interpolated by finding its containing triangle and interpolating from the vertices.

The algorithms for performing the triangulation and interpolation are extremely fast and the resection volume can be rapidly rendered seconds once outline demarcation is complete. However, the manual outlining process is tedious and timeconsuming. Seven algorithms for automatic prostate recognition in ultrasound from the literature are currently being assessed [4-12], however no algorithm has been found which performs faster than manual outlining (many are much slower), and all 
the algorithms fail on images which do not show the boundary with high clarity. This is particularly problematic since the available ultrasound machine (B\&K Medical, Model 1849) is old and produces very poor images when compared to the state-of-the art.

\section{Per-operative Ultrasound}

Work is underway to assess the potential of per-operative ultrasound for motion and deformation tracking. In the laboratory, a phantom has been made with a very clear boundary between prostate and non-prostate regions. Figure 11 shows an ultrasound image of the phantom taken in a water bath, and without insertion of a resectoscope. The boundary of the capsule and the urethra cavity are clearly visible. Automated recognition of the phantom boundary, within images captured from the available ultrasound equipment, is feasible. It is expected that image processing and model building time will be short enough to allow rapid update of the computer model as the phantom deforms.

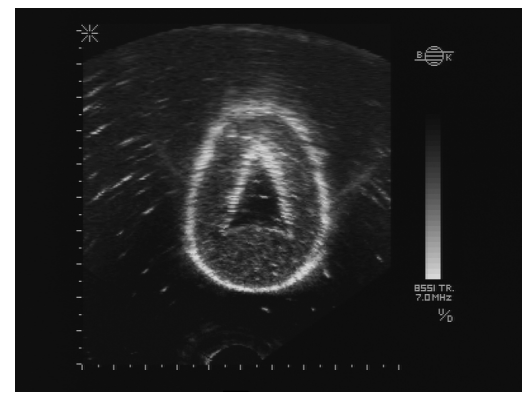

Fig. 11. Ultrasound image of the gelatine phantom taken in a water bath

A motorised holder to traverse the ultrasound probe has been designed so that the phantom may be continually imaged across the region of interest (the resection volume) during the TURP. The holder has one manual linear axis and one manual angular axis so that the probe may be positioned and locked to lie along the direction of the rectum. The motorised axis allows movement of the probe along the rectum using a stepper motor and lead screw under microprocessor control. The ultrasound probe is first clamped to the holder and registered with the optical tracking system by an IRED arrangement on the probe giving initial position and orientation of the probe. The position of the probe during resection can be obtained through the optical tracking system, or by using the initial position and calculating position by counting the steps of the motor. The latter method may be used in the operating theatre where line of sight to the IREDs on the probe will be obstructed.

A preliminary trial of per-operative ultrasound using a stationary probe without positional information has been conducted. Figure 12 shows the prostate outline and slight shadowing caused by the resectoscope within the image. Figure 13 shows the resected cavity with a much brighter edge than the prostate capsule. Figures 14 and 15 show the artefacts introduced when coagulating and cutting respectively.

The shadow caused by the resectoscope does not completely hide the section of prostate boundary behind it. Since the positions of the resectoscope and ultrasound probe within the scan are known, it is possible to anticipate the shadowing and tailor 
boundary detection methods accordingly. The artefacts caused by current through the resectoscope are far more problematic - indeed they occur at the time when it is crucial to track the prostate's movement. One possibility for overcoming this problem is to momentarily stop the current whilst an image is grabbed, then resume the cutting whilst the ultrasound probe is moved to the next acquisition position.

The cutting and coagulation artefacts do not pose a problem on the gelatine phantom, since resection of the phantom is performed without the current switched on.

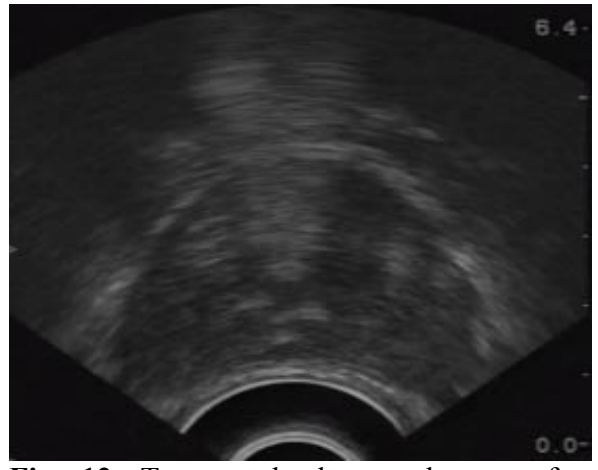

Fig. 12. Transrectal ultrasound scan of a human prostate, showing the shadow cast by the resectoscope

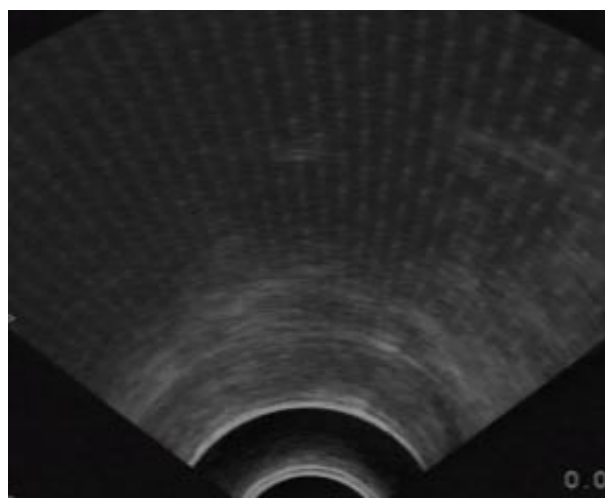

Fig. 14. Artefact whilst coagulating

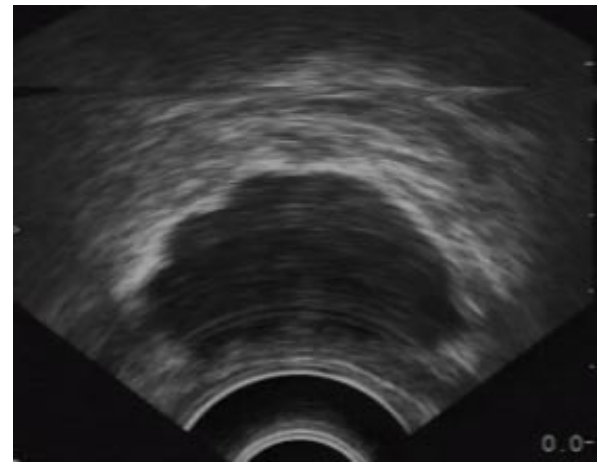

Fig. 13. The cavity formed (darker area roughly in the centre of the image) is easily identifiable

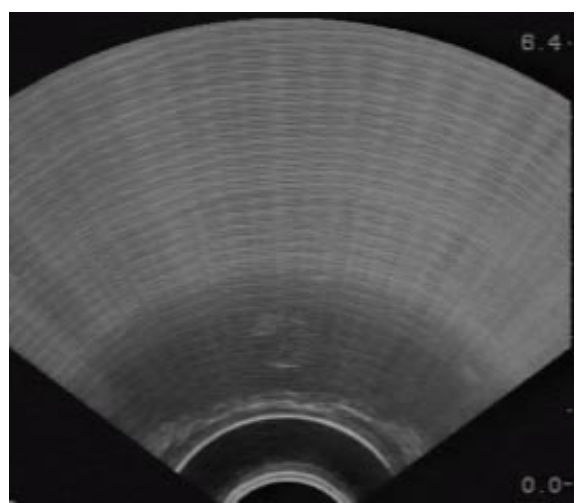

Fig. 15. Artefact whilst cutting

\section{Discussion and Conclusions}

Prostate phantoms which can be resected with a cutting loop and scanned with ultrasound are described. The procedure for using ultrasound to track prostate movement is presented. 
Assessment of tool mock-ups in the operating room has been carried out. This is the first step towards the evaluation of a computer-assisted surgical monitoring system for TURP.

A possible drawback is the difficulty for the surgeon to concentrate on the endoscopic view and also on the computer display simultaneously. The refresh rate needs to be real-time enough for any prostate movement during resection to be detected and compensated for. Line of sight between the optical tracker and the IRED tools needs to be maintained.

The monitoring system can both be used as an assessment and computer-based certification tool and it has scope for surgery planning and as a training tool.

\section{Acknowledgements}

The authors would like to thank Mr. Timoney and Mr. Kumar, Southmead Hospital, UK, the Engineering and Physical Sciences Research Council (EPSRC) of the UK, Limbs \& Things (UK), and Karl Storz Endoscopy (UK).

\section{References}

1. Gomes, M.P.S.F. and Davies, B.L. (2000) Computer-Assisted TURP Training and Monitoring, Medical Image Computing and Computer-Assisted Intervention MICCAI'00, Pittsburgh, USA, 669-677

2. Blandy, J. (1983) Benign Prostatic Hypertrophy, Springer-Verlag, New York

3. Bude, R.O. and Adler, R.S. (1995) An easily made, low-cost, tissue-like ultrasound phantom material, Journal of Clinical Ultrasound 23, 271-273

4. Aarnick, R., Geisen, R., Huynen, A., Rosette, J., Debruyne, F. and Wijkstra, H. (1994) A practical clinical method for contour determination in ultrasonographic prostate images, Ultrasound Med Biol 20 (8), 705-717

5. Arambula-Cosio, F. and Davies, B. (1999) Automated prostate recognition: a key process for clinically effective robotic prostatectomy, Med Biol Eng Comput 37, 236-243

6. Richard, W. and Keen, C. (1996) Automated texture-based segmentation of ultrasound images of the prostate, Comput Med Imag Graph 20 (3), 131-140

7. Prater, J. and Richard, W. (1992) Segmenting ultrasound images of the prostate using neural networks, Ultrasonic Imaging 14, 159-185

8. Richard, W., Grimmel, C., Bedigian, K. and Frank, K. (1993) A method for threedimensional prostate imaging using transrectal ultrasound, Comput Med Imag Graph 17(2), 73-79

9. Aarnick, R., Pathak, S., Rosette, J., Debruyne, F., Kim, Y. and Wijkstra, H. (1998) Edge detection in prostatic ultrasound images using integrated edge maps, Ultrasonics 36, 635642

10. Liu, Y., Ng, W., Teo, M. and Lim, H. (1997) Computerised prostate boundary estimation of ultrasound images using radial bas-relief method, Med Biol Eng Comput 35, 445-454

11. Levienaise-Obadia, B. and Gee, A. (1999) Adaptive segmentation of ultrasound images, Imag Vision Comput 17, 583-588

12. Crivianu-Gaita, D., Miclea, F., Gaspar, A., Margineatu, D. and Holban, S. (1997) 3D reconstruction of prostate from ultrasound images, Int J Medical Informatics 45, 43-51 